\title{
Metodología cualitativa en bibliotecología y ciencia de la información. Un análisis bibliográfico de artículos académicos
}

\author{
Patricia Hernández Salazar*
}

Artículo recibido:

17 de septiembre de 2018

Artículo aceptado:

20 de noviembre de 2018

Artículo de investigación

\section{Resumen}

El seguimiento del enfoque cualitativo para realizar investigaciones en las disciplinas bibliotecológica y de la información ha sido escaso. Una posible causa es su esencia basada en la relación directa con los sujetos/ objetos de estudio y en las implicaciones para los investigadores en cuanto a la cantidad de datos y su adecuada interpretación y narración. El objetivo del presente artículo es establecer la situación que prevalece sobre la aplicación de métodos cualitativos en bibliotecología y ciencia de la información. El cumplimiento de este objetivo requirió abordar tres temas principales: la esencia del enfoque cualitativo, las implicaciones de seguirlo y un panorama de su empleo, mediante

* Instituto de Investigaciones Bibliotecológicas y de la Información, UNAM, México

$$
\text { phs75599@unam.mx }
$$

INVESTIGACIÓN BIBLIOTECOLÓGICA, vol.33, núm.78, enero/marzo, 2019, México,
ISSN: 2448-8321 pp. 105-120 
un análisis bibliográfico de artículos académicos. El resultado refuerza la idea de que hay poco interés por realizar estudios cualitativos.

Palabras clave: Metodología cualitativa; Bibliotecología; Ciencia de la información; Implicaciones

Qualitative methodology in librarianship and information science: a bibliographic analysis of academic articles

Patricia Hernández Salazar

\begin{abstract}
The follow-up of the qualitative approach to carry out research in the librarianship and information disciplines has been up to now scarce. A possible cause is its essence based on a direct relationship with the subjects/objects of study, and on the implication for researchers regarding the amount of data and its adequate interpretation and narration. The purpose of the present paper is to establish the situation prevailing in respect to the application of qualitative methods in librarianship and information science. The fulfillment of this objective required to deal with three main issues: the essence of the qualitative approach, the implication of following it and an examination of its use, through a bibliographic analysis of academic papers. The result reinforces the idea that there is little interest in qualitative studies.
\end{abstract}

Keywords: Qualitative Methodology; Librarianship; Information Science; Implications

\title{
INTRODUCCIÓN
}

Ci se acepta que el objeto de estudio de la bibliotecología y la ciencia de la Oinformación es la relación entre la información y los sujetos que la utilizan, entonces una proporción considerable de investigaciones tendría que abordar problemas vinculados con personas; el mejor camino para resolver 
este tipo de problemas es mediante la metodología cualitativa, lo que nos llevaría a afirmar que en estas disciplinas se está siguiendo esta metodología, supuesto que no puede validarse cabalmente ya que en términos generales se ha empleado el enfoque cuantitativo.

Se deben realizar estudios que registren, interpreten y describan las palabras de los usuarios, se requiere la descripción narrativa de sus palabras: necesidades, inquietudes, comportamientos, satisfacciones y transformaciones cognitivas (utilización) con relación a la información.

Algunos autores ubican el primer estudio cualitativo en el siglo XIX (Taylor, Bogdan y DeVault, 2016), la mayoría coincide en que aparece formalmente en la segunda década del siglo XX, es decir, hace casi un siglo. Sin embargo, la investigación cualitativa por parte de los profesionales e investigadores de las disciplinas bibliotecológicas y de la información es escasa (Cook y Farmer, c2011; Goodman, 2011). En los textos que tocan el tema de manera genérica y mencionan algunas disciplinas que lo han seguido, nunca aparecen bibliotecología, ciencia de la información o afines.

Para apuntalar esta afirmación el propósito del presente artículo es establecer la situación que prevalece sobre la aplicación de métodos cualitativos en bibliotecología y ciencia de la información. El cumplimiento de este objetivo requirió abordar tres temas principales: la esencia del enfoque cualitativo, las implicaciones de seguirlo y un panorama de su empleo. Este último se desarrolló mediante el análisis de artículos publicados en la revista Investigación Bibliotecológica: archivonomía, bibliotecología e información, publicada por el Instituto de Investigaciones Bibliotecológicas y de la Información durante el periodo comprendido entre 2001 y marzo de 2018.

Es pertinente aclarar que no es la intención hacer generalizaciones, los resultados pretenden mostrar una forma de acercamiento al estado del arte sobre el uso de la metodología cualitativa para resolver problemas bibliotecológicos y de la información.

\section{ESENCIA DEL ENFOQUE CUALITATIVO}

Los primeros estudios que se basaron en el decir directo de los individuos se realizaron en el siglo XIX, por Frederick Le Play y Emile Durkheim (Taylor, Bogdan y DeVault, 2016); específicamente nombrados como metodología o investigación cualitativa fue en las primeras décadas del siglo XX por el grupo de Sociología de la Universidad de Chicago (entre 1910 y 1920). Los miembros de este grupo estudiaron comunidades urbanas, de delincuentes juveniles e inmigrantes polacos, lo que les permitió afirmar que en la vida 
social una constante son los cambios, lo que importa es poner en el centro de la investigación las interacciones, los procesos sociales y el punto de vista de los actores y narrarlos. Emplearon diversos métodos y técnicas, como la observación participante, entrevistas a profundidad y documentos personales (Strauss, 1987).

Según Seale et al. (2004), en una revisión hecha al catálogo SAGE ${ }^{1}$ en las últimas décadas ha aumentado exponencialmente el número de textos que tocan el tema de métodos cualitativos, de 10 en el periodo comprendido entre 1980 y 1987 a más de 130 entre 1995 y 2002. Se infiere que proyectivamente se ha seguido incrementando.

Un asunto que ha tenido gran atención es la delimitación conceptual del enfoque cualitativo, su esencia y caracterización; existe una buena cantidad de documentos que tratan el tema, aunque aparecen elementos comunes es más recurrente encontrar diferencias que dificultan la aprehensión puntual de su sentido y formas para seguirlo, por lo que se consideró conveniente abordarlo.

Se parte del término base cualidad, que Aristóteles explica ampliamente. Una aproximación conceptual aparece en su obra Categorías, la entiende como "[aquello] por lo que se dice [que los hombres] son tales o cuales" (2009: 86). Le concede varios sentidos que denomina especies, tres de los cuales son propiedades vinculadas con seres humanos: el primero se refiere al hábito (conducta estable y duradera) y a la disposición (conducta variable, acciones); el segundo es la capacidad o incapacidad natural que posee (aptitud), y el tercero alude a los afectos y afecciones (actitudes). Como se puede apreciar estas especies son características privativas de lo humano, con lo que se va dibujando lo esencial de la metodología cualitativa.

El término metodología nos lleva a ubicar las tendencias desde las cuales se han resuelto problemas de investigación y se relaciona con el paradigma. ${ }^{3}$ De acuerdo con Taylor, Bogdan y DeVault (2016) en las disciplinas sociales y humanísticas han dominado dos paradigmas, el positivismo y la fenomenología. El positivismo privilegia lo que es positivo, entendido como lo cierto o verdadero, lo efectivo; las disciplinas que siguen este paradigma tienen sus base en hechos objetivos o realidades concretas (tangibles), que sean percibidas mediante los órganos de los sentidos (Ferrater Mora, 2001), "busca los hechos o causas de los fenómenos sociales fuera de los estados subjetivos individuales”

Editorial creada en 1965 , publica contenidos innovadores y de alta calidad relacionados con la investigación y la enseñanza. https://us.sagepub.com/en-us/nam/home

2 Las palabras entre corchetes son aportación del traductor.

3 Se concibe al paradigma como un modelo que las comunidades epistémicas de un campo disciplinario siguen para crear conocimiento; integra problemas, principios teóricos y metodológicos. 
(Taylor, Bogdan y DeVault, 2016: 3); desde esta postura los científicos sociales miran los fenómenos colectivos como cosas o sucesos que ejercen una influencia externa sobre las personas.

La fenomenología "[...] consiste en reconsiderar todos los contenidos de conciencia. En vez de examinar si tales contenidos son reales o irreales, ideales, imaginarios, etc., se procede a examinarlos en cuanto son puramente dados. [...] No hay contenidos de conciencia, sino únicamente 'fenómenos' [...] es una pura descripción de lo que se muestra por sí mismo [...]" (Ferrater Mora, 2001, v. 2: 1240). Es un paradigma de corte descriptivo que intenta "entender los fenómenos sociales desde la perspectiva propia de los actores y examinar como el mundo es experimentado" (Taylor, Bogdan y DeVault, 2016:3).

Existen diferencias en los tipos de realidad social que se aspira conocer, los problemas que se pretende resolver, las preguntas que plantean esos problemas y sus respuestas, se utilizan formas y caminos para responderlas a través de los sujetos que representan esas realidades. El positivismo tiende a seguir un modelo de ciencia natural, busca causas y datos que puedan ser manejables sólo numéricamente con análisis estadísticos, mientras que la posición fenomenológica busca "entender en un nivel personal los motivos y creencias detrás de las acciones de la gente" (Taylor, Bogdan y DeVault, 2016: 4), se privilegian los significados y las interpretaciones de esos significados. Concretamente, "La frase metodología cualitativa se refiere en su sentido más amplio a la investigación que produce datos descriptivos de las propias palabras escritas o habladas de las personas y de su comportamiento observable" (Taylor, Bogdan y DeVault, 2016: 7). Su objetivo último es "[...] entender y representar las experiencias y acciones de la gente tal como ellos se encuentran, comprometen, y viven a través de situaciones. [...] el investigador intenta desarrollar entendimientos del fenómeno bajo estudio, basado tanto como sea posible en la perspectiva de aquellos seres que están siendo estudiados" (Elliott, Fischer y Rennie, 1999: 216).

Como principios se pueden apuntar los siguientes:

1. Importa el sentido que las personas le dan a las cosas y acontecimitos de sus vidas. Los investigadores empatizan y se identifican con la gente que estudian con el fin de entender la forma en la que perciben las cosas. Deben poner entre paréntesis las aproximaciones/suposiciones, preconcepciones y presuposiciones que tienen de la vida y del fenómeno que están estudiando.

2. Es de corte inductivo. Se desarrollan conceptos internos, producto del análisis de los propios datos recogidos, para entender y describir patrones. 
3. Se mira el escenario social y a la gente de manera holística, no se reducen a categorías o variables separadas, se relacionan como un todo. Se considera el contexto (situacional y estructural), y todo aquello que los hace ser y existir, como circunstancias pasadas y presentes.

4. Preocupa cómo piensa y actúa la gente en sus vidas diarias. Se debe interactuar con la gente de forma natural y no intrusiva.

5 Se requiere considerar todas las perspectivas posibles.

6. La validez como cualidad indispensable. Una investigación válida debe ceñirse al fenómeno que verdaderamente se investiga, evitando sesgos, al permanecer cerca del mundo empírico se logra un margen muy estrecho entre los datos y lo que la gente dice y hace en la realidad de sus contextos y situaciones (Taylor, Bogdan y DeVault, 2016: 7-11).

Recapitulando, los atributos que definen la esencia de la metodología cualitativa son los siguientes: relación directa con los sujetos/objetos de estudio, importancia del contexto e historia de los sujetos, retomar e interpretar el sentido y significado que dichos sujetos confieren a sus palabras, y examinar cada fenómeno de forma holística.

Entre las disciplinas que han seguido esta metodología destacan sociología, geografía, ciencia política, psicología, enfermería, psicoterapia, educación, negocios, ciencias de la salud, teología, educación musical, desarrollo humano, negocios, trabajo social, arquitectura y comunicación. Sobre todo, se asocia directamente con la antropología y la etnografía por el tipo de producto que deriva de su seguimiento, un texto narrativo que describe e interpreta las situaciones observadas.

\section{IMPLICACIONES DE LA INVESTIGACIÓN CUALITATIVA}

Los aspectos esenciales del enfoque cualitativo dan una idea de los compromisos y las repercusiones para los investigadores. Lo cualitativo se vincula con un marco conceptual fundamentado en las imágenes del mundo que poseen tanto los sujetos de estudio como de las personas que los estudian (Grover y Glazier, 1985). Para obtener un alto grado de objetividad el investigador debe dejar de lado las propias perspectivas y percibir las cosas como si fuera la primera vez que las viera. Los fenómenos y problemas son de corte humano, con la clara intención de abordarlos directamente desde y con las personas.

La recolección de datos privilegia la aplicación de instrumentos que impliquen sus voces (entrevistas personales o grupales, registros de observaciones directas y documentos personales, entre otros). Se utilizan datos 
asentados, escritos de una considerable extensión, los documentos orales deberán grabarse en algún dispositivo para que puedan reproducirse y transcribirse para tener el panorama completo de las situaciones de los sujetos y claridad del orden que se seguirá para recuperarlos, analizarlos e interpretarlos. Esta etapa se hace complicada, requiere de una gran disposición, paciencia y sistematización.

Los aspectos que revisten un mayor compromiso son los vinculados con el análisis y la interpretación de datos, pues si bien pueden considerarse como un proceso fusionado existen algunos elementos privativos de cada uno. La fase de análisis permite ir perfilando el sentido de los datos, la dificultad radica en que "el proceso no es fundamentalmente mecánico o técnico; es un proceso inductivo de razonamiento, pensamiento y teorización” (Taylor, Bogdan y DeVault, 2016: 169). Su objetivo es obtener un entendimiento profundo de los fenómenos y refinar su interpretación, descubrir temas, desarrollar conceptos, establecer relaciones entre los conceptos, proposiciones y patrones. Las acciones que se realizan son codificación y reducción, se crean códigos (abreviaturas o símbolos) que representan categorías, se anotan en frases o párrafos, de esta manera se reducen los datos, se reestructuran, se extraen y se descubren relaciones.

Con la codificación y reducción se logra la representación condensada de categorías, tipologías y proposiciones, se requieren muchas horas de trabajo. Reducir comprende:

1. Leer y releer tus datos.

2. Seguir corazonadas, interpretaciones e ideas.

3. Buscar temas que aparezcan frecuentemente.

4. Construir tipologías.

5. Desarrollar conceptos y proposiciones teóricas. [...]

7. Elaborar mapas, diagramas, y figuras.

8. Escribir memorandos analíticos. (Taylor, Bogdan y DeVault, 2016: 171)

En cuanto a la interpretación, "[...] exige una reflexión sistemática y crítica de todo el proceso de la investigación, con el fin de contrastarlo, por un lado, con la teoría y, por el otro, con los resultados prácticos. Se trata de verificar en la investigación la adecuación entre objeto y pensamiento" (Pérez Serrano, c2001, v. 2: 127). La interpretación es un acto reflexivo (poner atención) en relación con el problema de representación de la realidad y la pretendida autoridad sobre el manejo del tema, integrar e interpretar es la fase más difícil pues requiere una gran capacidad de creación y generación de ideas nuevas, permite al investigador "comprobar si las respuestas que ha encontrado 
funcionan o no en la práctica” (Pérez Serrano, c2001, v. 2: 128). Se significa y describe lo dicho por las personas, lo cual es el resultado de vincular cinco elementos:

$$
\begin{gathered}
\text { Principio - Marco teórico general - Problema/fenómeno } \\
\text { de estudio - Propósito - Método }
\end{gathered}
$$

Otro aspecto que requiere gran atención es la escritura o narración de los resultados. No sólo se reportan o describen escenarios, se crean miradas particulares de una parte de la realidad de los sujetos, lo que implica un gran manejo de la escritura (sintaxis y ortografía). De esto surge la importancia de la forma narrativa en la que se representan los datos recogidos, debe mostrar riqueza descriptiva, proveer significados a los detalles cercanos del contexto dentro del cual se dan los eventos y situaciones que viven las personas cotidianamente.

Los reportes o escritos producto de un estudio cualitativo deben contener los siguientes apartados: declaración del problema, marco teórico, camino metodológico seguido, naturaleza y número de escenarios e informantes, datos de identidad, relación con la gente o comunidad de estudio, tiempo y duración, forma de analizar e interpretar los datos, y suposiciones o marco de pensamiento propio.

Una condición imprescindible en los investigadores cualitativos es la integridad, en relación con las personas y con las cosas; deben evitar involucrarse al grado de sesgar los resultados, salirse del entorno investigativo y presentar la totalidad de los hallazgos (Marshall y Rossman, c2016).

Lo íntegro también se vincula con los términos escrupuloso, rigurosidad y respeto. No usar a las personas como fin propio, concederles el derecho a decidir su participación y garantizar su privacidad y anonimato.

En suma, las características que debe tener un investigador cualitativo son integridad, creatividad, intuición, sensibilidad, autenticidad, credibilidad, receptividad y flexibilidad; que disfrute el trabajo con una gran cantidad de datos, busque la reciprocidad, evite el sesgo, que sea objetivo y constante en la generación de conclusiones.

\section{Panorama de la APliCACión Del ENFoque CUALITATIVO EN} BIBLIOTECOLOGÍA, CIENCIA DE LA INFORMACIÓN Y DISCIPLINAS AFINES

La metodología en estas disciplinas ha seguido el paradigma hegemónico determinado por las ciencias naturales (y en general por la producción del 
conocimiento) y por algunas disciplinas sociales, el positivismo. La intención ha sido convertirse en ciencias y ser reconocidas a partir de utilizar este paradigma y los métodos y técnicas cuantitativas, lo positivo y numérico valida una existencia científica. Sin embargo, el objeto de estudio -la relación entre información y sujeto-, los fenómenos de investigación derivados de personas y sus contextos personales y sociales deberían ver como una causalidad la aplicación del enfoque cualitativo.

De acuerdo con Evans (1976), la fecha más lejana en la que se utilizó un camino cualitativo en bibliotecología fue en 1896 en un estudio hecho por Mary Cutler, quien pretendió identificar el espíritu de la vida de los sujetos y relacionarlos con la biblioteca, lo cual posteriormente se inscribió dentro de los estudios de comunidad. Integró elementos que la pusieron en contacto con actividades diarias de las personas y sus requerimientos hacia la biblioteca, el contexto de sus actividades cotidianas y el significado de sus propias acciones.

Se hizo una búsqueda exhaustiva sobre estudios que trataran el estado del arte del uso de la metodología cualitativa, el resultado arrojó sólo trabajos anglosajones. Gauchi Risso (2017) examinó diversas publicaciones (Library and Information Science Abstracts-LISA, Social Science Citation Index-SSCI y de organismos como la International Federation of Library Associations-IFLA), con el fin de determinar el estado de la cuestión de la metodología en bibliotecología, clasificó los resultados en métodos cuantitativos y cualitativos, consideró tesis doctorales y artículos; para este trabajo sólo se tomaron en cuenta los datos de los artículos de corte cualitativo. A continuación se muestran los resultados, los cuales están agrupados por periodos; se anota el porcentaje y el método o la técnica utilizados:

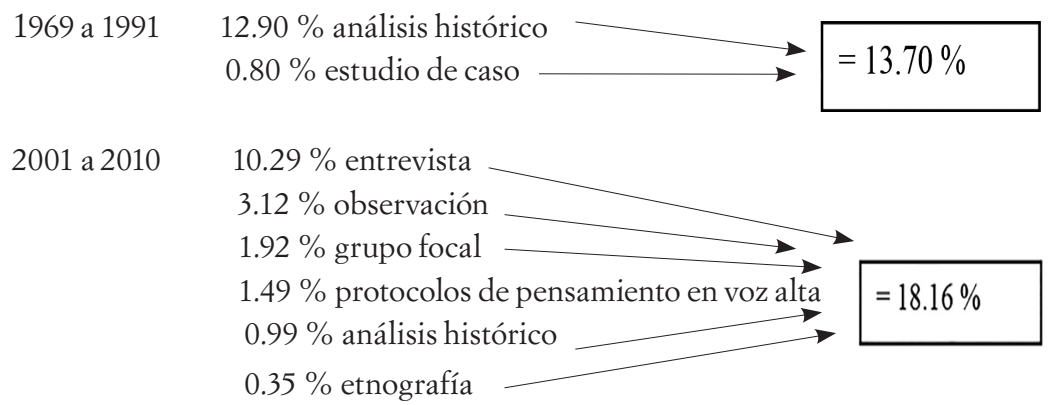

Los datos muestran un reducido número de artículos relacionados con la metodología cualitativa (menos de una quinta parte), en el periodo más anterior (1969 a 1991) hay un porcentaje mayor del análisis histórico. Es posible inferir que se buscaban las bases históricas de la disciplina y eso motivó realizar este tipo de investigaciones. Sobresale que en la primera década de este siglo se haya ampliado la variedad de métodos y técnicas de dos a seis. 
Estas cifras confirman lo expresado en párrafos anteriores, que en las disciplinas bibliotecológicas y de la información hay poca inclinación hacia el enfoque cualitativo, a pesar de que un elemento fundamental de su objeto de estudio es la persona y su agrupación en comunidades.

En un examen sobre la transformación metodológica para abordar el campo fenoménico de usuarios de la información (Hernández Salazar, 2014) se encontró que a partir de la década de los setenta empieza un cambio en la naturaleza de la investigación, del diseño de sistemas de información basado en el paradigma físico al paradigma cognitivo centrado en el usuario con autores como Brookes, Belkin, Ingwersen, Kuhlthau, Wilson y Dervin, por lo que se inicia la aplicación de métodos y técnicas cualitativas. En los setenta fue el incidente crítico, para los ochenta ya se encuentran entrevista a profundidad, análisis del discurso, grupo focal y estudio de caso; en los noventa se agrega el análisis del discurso y el sense-making; ${ }^{4}$ en la primera década del siglo XXI historias de vida, fenomenografía y teoría fundamentada.

Con el fin de establecer la situación que prevalece en Iberoamérica, se analizaron los artículos publicados en la revista Investigación Bibliotecológica: archivonomía, bibliotecología e información ${ }^{5}$ del Instituto de Investigaciones Bibliotecológicas y de la Información durante el periodo comprendido entre 2001 a marzo de 2018. La elección del periodo permite dar continuidad a los rangos estudiados y de la revista por su cobertura regional y su clasificación en el rango Q2.

El análisis se hizo en dos etapas, una primera correspondió a la mera búsqueda y recuperación cuantitativa, en la segunda se hizo la lectura de cada artículo para validar si realmente se trataba de investigación cualitativa desde los elementos esenciales e implicaciones explicadas en los apartados anteriores.

Primera etapa. Los artículos publicados entre 2001 y marzo de 2018 fueron 369. Se hicieron algunas búsquedas restringidas a frases completas, por ejemplo, enfoque cualitativo, métodos cualitativos o metodología cualitativa y el resultado fue 0 , por lo que se determinó que los descriptores de ciertas frases aparecieran juntos o separados en cualquier campo. En el caso de los métodos y técnicas se decidió delimitar por frases exactas para que los resultados fueran pertinentes, el total de registros fue 168, lo que correspondió al $46 \%$. La distribución se muestra en la Tabla 1:

4 Para abundar sobre el sense-making véase Rendón Rojas y Hernández Salazar (2010).

5 Revista científica arbitrada de corte internacional, publica resultados científicos originales e inéditos, su cobertura es de 1986 hasta la fecha, su periodicidad es trimestral. Está clasificada en el Q2 dentro de los sistemas internacionales y nacionales que generan indicadores de impacto y visibilidad. Disponible en http://rev-ib.unam.mx/ib/index.php/ib/index 


\begin{tabular}{|l|r|}
\hline \multicolumn{2}{|c|}{ Descriptores en cualquier campo } \\
\hline Investigación cualitiativa & 41 \\
\hline Metodología cualitativa & 30 \\
\hline Métodos cualitativos & 26 \\
\hline Enfoque cualitativo & 22 \\
\hline \multicolumn{1}{|c|}{ Frases exactas por tipo de método o técnica } \\
\hline Estudio de caso & 18 \\
\hline Focus group & 8 \\
\hline Análisis del discurso & 6 \\
\hline Etnografía & 4 \\
\hline Grupo focal & 4 \\
\hline Historias de vida & 1 \\
\hline Entrevista a profundidad & 1 \\
\hline Historia oral & 1 \\
\hline Incidente crítico & 1 \\
\hline Sense-making & \\
\hline Teoría fundamentada & \\
\hline
\end{tabular}

Tabla 1. Distribución de métodos y técnicas cualitativas

Se cruzaron los resultados con el fin de eliminar los registros repetidos, fueron 73; también se descartaron los comentarios o reseñas cuya cifra fue 14 , al restarlos la cantidad quedó en 81 (22\%).

Segunda etapa. Se leyeron los 81 artículos para determinar si efectivamente su contenido estaba relacionado con la metodología cualitativa, esto debido a que algunos títulos mostraban su evidente vinculación con el enfoque cuantitativo. No se consideró la metodología mixta, pues lo que se quiere resaltar es la tendencia cualitativa desde lo fenomenológico, acercamiento directo con los sujetos e interpretación de sus experiencias vivenciales y expresiones orales. La lectura y examen puntual permitió establecer que sólo 21 artículos eran estudios cualitativos, es decir, únicamente el $6 \%$ de la totalidad (369) de los artículos publicados durante el periodo de estudio. Las causas por las que se excluyeron los documentos se mencionan a continuación:

- Los términos o frases aparecían en el estado del arte del tema en cuestión, en alguna cita o título de la lista de obras consultadas (bibliografía o referencias).

- Textos de corte genérico que sugerían seguir métodos, metodología o enfoque cualitativo. 
- Lo cualitativo se mencionaba como calificativo de diversos términos: evaluación, salto, cambio, tipo, fuentes musicales y clasificación, entre otros.

- Considerar en estudios cuantitativos indicadores cualitativos porque aluden a la calidad (enlaces de correlación en sitios web mediante el uso de softwares como Linkbot; criterios para examinar revistas como identidad editorial, de revisión por pares, y metodológicos o de estructura) o porque -según los autores- son medidas estadísticas más elaboradas (por ejemplo, probabilidad y correlación).

Lo anterior permite establecer que existe una gran confusión en cuanto a lo cualitativo y el uso indiscriminado del término por la falta de claridad de su esencia e implicaciones. Sobresale que refieren lo cualitativo a aspectos de calidad y evaluación, los autores recomiendan apoyar los resultados cuantitativos con datos o indicadores "de calidad" o cualitativos.

Para analizar los 21 artículos se establecieron cinco categorías de estudio: año, país, área de investigación, carácter del contenido (teórico, aplicado o análisis bibliográfico), y método o técnica utilizados (Tabla 2).

\begin{tabular}{|c|c|l|c|l|}
\hline Año & País & \multicolumn{1}{|c|}{ Área de investigación } & $\begin{array}{c}\text { Carácter del } \\
\text { contenido }\end{array}$ & \multicolumn{1}{|c|}{ Método/Técnica } \\
\hline 2001 & México & Evaluación del aprendizaje & Aplicado & Observación participante \\
\hline 2002 & España & Análisis y evaluación-investigación & AB $^{*}$ & \\
\hline 2005 & $\begin{array}{c}\text { Vene- } \\
\text { zuela }\end{array}$ & Usuarios-constructivismo & Teórico & \\
\hline 2008 & México & Lectura & AB & \\
\hline 2008 & España & Grupos de Investigación & Teórico & \\
\hline 2008 & México & $\begin{array}{l}\text { Alfabetización informativa-apropia- } \\
\text { ción de habilidades }\end{array}$ & Aplicado & $\begin{array}{l}\text { Etnografía de la } \\
\text { comunicación }\end{array}$ \\
\hline 2008 & Brasil & Alfabetización informativa-cognición & Aplicado & $\begin{array}{l}\text { Observación participante } \\
\text { Intervención educativa }\end{array}$ \\
\hline 2010 & México & Evaluación de bibliotecas digitales & AB & \\
\hline 2011 & Cuba & Alfabetización informativa-evaluación & Aplicado & Investigación-acción \\
\hline 2014 & España & Estudio de usuarios & AB & \\
\hline 2014 & España & $\begin{array}{l}\text { Educación bibliotecológica-comuni- } \\
\text { dades de práctica }\end{array}$ & Aplicado & Estudio de caso \\
\hline 2014 & Colombia & Acreditación IES-autoevaluación & Aplicado & $\begin{array}{l}\text { Estudio de caso } \\
\text { Grupos de discusión }\end{array}$ \\
\hline 2014 & España & Procesos cognitivos & Aplicado & $\begin{array}{l}\text { Entrevista a profundidad } \\
\text { Teoría fundamentada }\end{array}$ \\
\hline
\end{tabular}




\begin{tabular}{|c|c|c|c|c|}
\hline 2015 & Colombia & Competencias informativas & $A B$ & \\
\hline 2015 & Perú & Enseñanza universitaria & Aplicado & Estudio de caso \\
\hline 2015 & Colombia & $\begin{array}{l}\text { Competencias informativas-educa- } \\
\text { ción superior }\end{array}$ & Aplicado & Estudio de caso \\
\hline 2016 & México & $\begin{array}{l}\text { Bibliotecas públicas-evaluación } \\
\text { recursos humanos }\end{array}$ & Aplicado & $\begin{array}{l}\text { Observación directa } \\
\text { Observación participante }\end{array}$ \\
\hline 2017 & Brasil & $\begin{array}{l}\text { Mediación en la información } \\
\text { (modelo)-habilidades informativas }\end{array}$ & Aplicado & $\begin{array}{l}\text { Observación participante } \\
\text { Entrevista a profundidad } \\
\text { Grupo focal }\end{array}$ \\
\hline 2017 & España & Redes sociales & Aplicado & $\begin{array}{l}\text { Análisis de contenido/ } \\
\text { tuits }\end{array}$ \\
\hline 2017 & Colombia & $\begin{array}{l}\text { Competencias informativas-educa- } \\
\text { ción superior }\end{array}$ & Aplicado & Estudio de caso \\
\hline 2017 & Ecuador & Preservación-material audiovisual & Aplicado & $\begin{array}{l}\text { Observación no } \\
\text { participante }\end{array}$ \\
\hline
\end{tabular}

Tabla 2. Resultados del análisis de los artículos ${ }^{*} A B$-análisis bibliográfico

No existe un aumento numérico consistente en cuanto al año de publicación, puesto que en 2008 fueron 4, se creería que en los años siguientes habría más; sin embargo, en 2010 y 2011 sólo hubo uno; nuevamente en 2014 se publican 4; en 2015, 3, y en 2017, 4. Estos datos no permiten aventurar una tendencia.

Respecto a la categoría país, resalta que en 8 se está trabajando con este enfoque, si se mira el mapa de América del Sur es posible afirmar que 5 tienen una cercanía geográfica. El país que más ha generado artículos es España con 6, le sigue México con 5, Colombia con 4, Brasil con 2, y Cuba, Ecuador, Perú y Venezuela han publicado 1.

Como áreas disciplinarias aparecen varias que pueden integrarse en 5, cabe mencionar que en algunos artículos se tocaba más de un tema:

- Alfabetización informativa ${ }^{6} 33 \%$ (7 artículos). Operaciones cognitivas para desarrollar la capacidad lectora, formación de mediadores, estrategias de mediación, modelos para desarrollar y evaluar competencias/habilidades informativas (informacionales) en alumnos de instituciones de educación primaria y superior, y creencias y adhesiones en la formación de competencias informativas.

6 La alfabetización informativa es entendida como la "acción educativa sistematizada destinada a proveer a los sujetos de un conjunto de habilidades, procesos de pensamiento, como el pensamiento crítico, y actitudes que le permitan acceder, evaluar y usar efectivamente la información, para cubrir una necesidad dada. Esta acción deberá promover que aprendan a aprender y generar aprendizajes para toda la vida” (Hernández Salazar, 2012: 32), de aquí que se hayan incluido los temas relacionados con cognición, formación y habilidades informativas. 
- Usuarios $24 \%$ (5 artículos). Constructivismo, estudio de usuarios, procesos cognitivos, enfoque sociopsicológico, redes sociales y lectura.

- Evaluación 24 \% (5 artículos). Aprendizaje, investigación, colecciones, bibliotecas digitales y públicas (recursos humanos).

- Educación bibliotecológica 14 \% (3 artículos). Alternativas pedagógicas, evaluación, acreditación y comunidades de práctica,

- Sistemas de información 5 \% (1 artículo). Diseño, preservación, colecciones audiovisuales televisivas y sobre lectura y conocimiento.

En su mayoría son fenómenos referidos a sujetos, lo que muestra que los investigadores de estas áreas están conscientes de la importancia de seguir una metodología cualitativa que permita el acercamiento y el registro de sus necesidades y comportamientos desde sus voces. Sobresale que haya al menos un artículo sobre el diseño de sistemas de información, esto indica que empieza a haber conciencia de la importancia de considerar al individuo para diseñarlos.

Con referencia a la categoría carácter del contenido la mayoría de los artículos $67 \%$ (14) es aplicado, esto permitió identificar varios métodos y técnicas, algunos ya mencionados: observación (6), que se realizó de tres formas: directa, participante y no participante; estudio de caso (5), entrevista a profundidad (2), análisis de contenido (1), grupo focal (1), y teoría fundamentada (1). Surgieron otros: etnografía de la comunicación, intervención educativa, investigación-acción y grupos de discusión, los cuales fueron empleados una sola vez. Le siguen los análisis bibliográficos con $24 \%(5)$ y $9 \%$ de corte teórico (2).

Aunque en términos generales los fenómenos de estudio están vinculados con sujetos y los métodos y técnicas elegidos pueden incidir en cualquier área de investigación reportada, es posible establecer una relación entre éstos y el método o técnica utilizados. Logros educativos entre los que se puede incluir la alfabetización informativa son mirados de cerca, desde y con los sujetos, por lo que se aplican la observación y la etnografía. La evaluación, abordada de forma holística y participativa, se basó en la investigación-acción y en los estudios de caso. Por su parte, procesos y mediaciones cognitivas, que pueden presentar un alto grado de subjetividad, requirieron entrevistas a profundidad y teoría fundamentada.

Al conectar las categorías de país con área de investigación se identifican ciertas tendencias. En México el enfoque cualitativo se está aplicando en estudios relacionados (3) con la evaluación (de aprendizajes y bibliotecas), y en segundo plano con la alfabetización informativa y la lectura. Brasil y Colombia lo han trabajado básicamente en la alfabetización y competencias informativas. En España se ha aplicado este enfoque en diversas áreas, investigación, 
educación, procesos cognitivos, estudios de usuarios y redes. La vinculación entre año y área de investigación deja en claro que a partir de 2008 ha habido un gran interés por el tema de alfabetización informativa.

\section{CONSIDERACIONES FINALES}

Si en el objeto de estudio de la bibliotecología y la ciencia de la información aparece la persona que la usa, resulta fundamental establecer un contacto directo con ella; este contacto se posibilita al solucionar los problemas de investigación mediante la metodología cualitativa. Sin embargo, en estas disciplinas se ha seguido escasamente este enfoque, lo que se evidencia con los resultados obtenidos: de 369 artículos publicados en la revista Investigación Bibliotecológica: archivonomía, bibliotecología e información en el periodo comprendido entre 2001 a marzo de 2018, solamente 21 (6 \%) emplearon el enfoque cualitativo.

Las razones pueden ser múltiples, su esencia humanista y sus implicaciones, la formación de los recursos humanos que tiende a seguir un paradigma positivista de normalización y los supuestos que dicha normalización resuelve los problemas de los sujetos, sin considerarlos directamente. La práctica profesional que privilegia las estadísticas y no busca la explicación de dichos números, pues es la forma como las instituciones miden su trabajo.

Se recomienda a las instituciones que forman a los profesionales de la información integrar contenidos sobre la metodología cualitativa en las asignaturas de metodología y seminarios de investigación, así como instar a las entidades de las que dependen las unidades de información a crear formas de medición del trabajo bibliotecario de corte cualitativo.

Se prueba el supuesto sobre el poco uso de la metodología cualitativa por los investigadores de bibliotecología, ciencia de la información y disciplinas afines. Si bien en el campo usuarios de la información ya existen estudios cualitativos, habrá que motivar su utilización en otras áreas como organización de la información, educación y tecnologías de la información y comunicación, cuyo vínculo con sujetos es patente, y así obtener hallazgos más cercanos a la realidad social.

\section{REFERENCIAS}

Aristóteles. 2009. Categorías. Trad. de Eduardo Sinnott. Buenos Aires: Colihue.

Cook, Douglas y Lesley Farmer. c2011. Using qualitative methods in action research: how librarians can get to the why of data. USA: Association of College and Research Libraries. 
Elliott, Robert, Constance T. Fischer y David L. Rennie. 1999. "Evolving guidelines for publication of qualitative research studies in psychology and related fields". British Journal of Clinical Psychology 38: 215-229.

Evans, Charles. 1976. "A history of community analysis in American Librarianship". Library Trends 24 (3): 441-457. http://citeseerx.ist.psu.edu/viewdoc/download?doi=10.1.1.204.7464\&rep=rep1\&type $=$ pdf

Ferrater Mora, José. 2001. Diccionario de filosofía. Barcelona: Editorial Ariel. 4 vol.

Gauchi Risso, Verónica. 2017. "Estudio de los métodos de investigación y técnicas de recolección de datos utilizadas en bibliotecología y ciencia de la información”. Revista Española de Documentación Científica 40 (2): 1-13. doi: http://dx.doi. org/10.3989/redc.2017.2.1333

Goodman, Valeda Dent. 2011. Qualitative research and the modern library. Oxford, U.K.: Chandos Publishing, Woodhead Publishing Limited.

Grover, Robert y Jack Glazier. 1985. "Implications for application of qualitative methods to Library and Information Science research". Library and Information Science Research 7 (3): 247-260.

Hernández Salazar, Patricia. 2012. "Contexto teórico de la Alfabetización Informativa”, en Tendencias de la Alfabetización Informativa en Iberoamérica, coordinado por Patricia Hernández Salazar, 3-46. México: UNAM, Centro Universitario de Investigaciones Bibliotecológicas.

Hernández Salazar, Patricia. 2014. "Transformación de la metodología para estudiar a los usuarios de la información”, en Naturaleza y método de la investigación bibliotecológica y de la información, coordinado por Jaime Ríos Ortega y César Augusto Ramírez Velázquez, 209-252. México: UNAM, Instituto de Investigaciones Bibliotecológicas y de la Información.

Marshall, Catherine y Gretchen B. Rossman. c2016. Designing qualitative research. Los Ángeles, California: SAGE.

Pérez Serrano, Gloria. c2001. Investigación cualitativa. Retos e interrogantes. Madrid: Editorial La Muralla, S. A. 2 v.

Rendón Rojas, Miguel Ángel y Patricia Hernández Salazar. 2010. "Sense-making: ¿metateoría, metodología o heurística?”. Investigación Bibliotecológica: archivonomía, bibliotecología e información 24 (50): 61-81.

Seale, Clive, Giampietro Gobo, Jaber F. Gubrium y David Silverman. 2004. Qualitative research practice. London: SAGE.

Strauss, Anselm Leonard. 1987. Qualitative analysis for social scientists. Cambridge: Cambridge University Press.

Taylor, Steven J., Robert Bogdan y Marjorie L. DeVault. 2016. Introduction to qualitative research methods: a guidebook and resource. New Jersey, USA: John Wiley \& Son.

Para citar este texto:

Hernández Salazar, Patricia. 2019. "Metodología cualitativa en bibliotecología y ciencia de la información. Un análisis bibliográfico de artículos académicos". Investigación Bibliotecológica: archivonomía, bibliotecología e información 33 (78): 105-120. http://dx.doi.org/10.22201/iibi.24488321xe.2019.78.58024 